\title{
Effectiveness of pandemic and seasonal influenza vaccine in preventing pandemic influenza A(H1N1)2009 infection in England and Scotland 2009-2010
}

\author{
P Hardelid (pia.hardelid@hpa.org.uk) ${ }^{1}$, D M Fleming ${ }^{2}$, J McMenamin ${ }^{3}$, N Andrews ${ }^{1}$, C Robertson ${ }^{3,4}$, P SebastianPillai ${ }^{1}$, J Ellis ${ }^{1}$, \\ W Carman ${ }^{5}$, T Wreghitt ${ }^{6}$, J M Watson ${ }^{1}$, R G Pebody ${ }^{1}$ \\ 1. Health Protection Agency Centre for Infections, London, United Kingdom \\ 2. Royal College of General Practitioners Research and Surveillance Centre, Birmingham, United Kingdom \\ 3. Health Protection Scotland, Glasgow, United Kingdom \\ 4. University of Strathclyde, Glasgow, United Kingdom \\ 5. West of Scotland Specialist Virology Centre, Glasgow, United Kingdom \\ 6. Health Protection Agency Regional Microbiology Network, Cambridge, United Kingdom
}

Citation style for this article:

Hardelid P, Fleming DM, McMenamin J, Andrews N, Robertson C, SebastianPillai P, Ellis J, Carman W, Wreghitt T, Watson JM, Pebody RG. Effectiveness of pandemic and seasonal influenza vaccine in preventing pandemic influenza $A\left(\mathrm{H}_{1} \mathrm{~N}_{1}\right) 2009$ infection in England and Scotland 2009-2010. Euro Surveill. 2011;16(2):pii=19763.

Available online: http://www.eurosurveillance.org/ViewArticle.aspx?Articleld=19763

Following the global spread of pandemic influenza $A\left(H_{1} N_{1}\right) 2009$, several pandemic vaccines have been rapidly developed. The United Kingdom and many other countries in the northern hemisphere implemented seasonal and pandemic influenza vaccine programmes in October 2009. We present the results of a case-control study to estimate effectiveness of such vaccines in preventing confirmed pandemic influenza infection. Some 5,982 individuals with influenza-like illness seen in general practices between November 2009 and January 2010 were enrolled. Those testing positive on PCR for pandemic influenza were assigned as cases and those testing negative as controls. Vaccine effectiveness was estimated as the relative reduction in odds of confirmed infection between vaccinated and unvaccinated individuals. Fourteen or more days after immunisation with the pandemic vaccine, adjusted vaccine effectiveness (VE) was $72 \%$ (95\% confidence interval (CI): $21 \%$ to $90 \%$ ). If protection was assumed to start after seven or more days, the adjusted VE was $71 \%$ ( $95 \% \mathrm{Cl}: 37 \%$ to $87 \%$ ). Pandemic influenza vaccine was highly effective in preventing confirmed infection with pandemic influenza $A\left(\mathrm{H}_{1} \mathrm{~N}_{1}\right)_{2009}$ from one week after vaccination. No evidence of effectiveness against pandemic influenza $A\left(H_{1} N_{1}\right) 2009$ was found for the 2009/10 trivalent seasonal influenza vaccine (adjusted VE of $-30 \%$ ( $95 \% \mathrm{Cl}$ : $-89 \%$ to $11 \%)$ ).

\section{Introduction}

Following the emergence and rapid global spread of pandemic influenza $A\left(\mathrm{H}_{1} \mathrm{~N}_{1}\right)_{2009}$ virus in April 2009 [1], several vaccines against this virus were quickly developed [2-6]. Clinical trials, including products with a new squalene adjuvant (MF59 or $\left.\mathrm{AS}_{53}\right)$ demonstrated that these novel pandemic vaccines were immunogenic in various target populations [2-6]. Published work on the possible effect of prior trivalent seasonal influenza vaccination on the subsequent risk of pandemic influenza infection has been conflicting: some have suggested a protective effect [7], others have found no association [8-10], and recent work from Canada has reported an increased risk of subsequent pandemic infection [11].

The United Kingdom (UK), as many other countries in the northern hemisphere, implemented its seasonal and pandemic influenza vaccine programmes in autumn 2009. Two pandemic vaccines were introduced in the UK: Pandemrix (GlaxoSmithKline), an inactivated low-dose influenza vaccine with one dose containing 3.75 g haemagglutinin (HA) equivalent of the influenza A/California/7/2009 isolate combined with the $\mathrm{AS} 03$ adjuvant) and Celvapan (Baxter), a wholevirion, Vero cell-derived influenza vaccine with a dose of $7.5 \mu \mathrm{g}$ of influenza $\mathrm{A}\left(\mathrm{H}_{1} \mathrm{~N}_{1}\right) \mathrm{HA}$ antigen of the $\mathrm{A} /$ California/07/2009 isolate. The pandemic vaccine programme was initially targeted at clinical risk groups older than six months, pregnant women and healthcare workers [12] and later extended to all healthy children six months to five years of age. Pandemrix was the main vaccine administered through the UK pandemic vaccine programme: by late February 2010, provisional uptake for the first dose of Pandemrix in England was $37.1 \%$ for clinical at-risk groups, $20.4 \%$ for healthy children six months to five years of age and $39.9 \%$ for healthcare workers [13].

The UK has an established surveillance system to monitor the effectiveness of the annual seasonal influenza vaccine programme. The system uses routine epidemiological data generated through swabbing of cases of influenza-like illness (ILI) presenting in primary care in England and Scotland [14]. Using this approach, this study sets out to provide estimates of the effectiveness of the pandemic and seasonal influenza vaccine 
programmes in preventing infection with pandemic influenza $\mathrm{A}\left(\mathrm{H}_{1} \mathrm{~N}_{1}\right) 2009$.

\section{Methods}

Study population and period

This study uses data from three influenza sentinel surveillance schemes in England and Scotland: the Royal College of General Practitioners' surveillance scheme (RCGP) covers 96 practices and ca. 900,000 patients throughout England (65 practices contribute to the swabbing programme), the Health Protection Agency (HPA) Regional Microbiology Network (RMN) surveillance scheme includes 45 contributing general practices and covers around 400,000 patients, and the Health Protection Scotland (HPS) scheme covers 101 general practices and 640,931 patients in Scotland (90 practices contribute to swabbing).

In all three schemes, clinicians are instructed to provide nose and throat swabs from a convenience sample of patients presenting with acute onset of respiratory illness, i.e.rapid development of appropriate symptoms usually with fever. No particular age group is specifically targeted and swabbing is undertaken regardless of prior influenza vaccination status of the patient.

This study covers samples collected in the period from 1 November 2009 (the pandemic influenza vaccination programme was rolled out across the UK on the 21 October) to 29 January 2010.

Cases were defined as individuals presenting with ILI in one of the participating practices in the defined study period who were swabbed and tested positive for pandemic influenza $A\left(\mathrm{H}_{1} \mathrm{~N}_{1}\right) 2009$ by RT-PCR. Controls were individuals presenting with ILI in the same period who were swabbed and tested negative. If they tested positive for other non-influenza respiratory viruses they were still included in the control group. Individuals who tested positive for other subtypes of influenza $A$ or for influenza B were excluded from the vaccine effectiveness (VE) estimates.

A standard specimen request form provided demographic and clinical information on cases and controls including date of birth, gender, date of onset, date of specimen collection, influenza vaccination status and vaccination date. Information on type of vaccine and dose was also collected.

\section{Laboratory methods}

Samples were sent to the HPA Centre for Infections (RCGP scheme), local HPA Regional Microbiology Network laboratories (RMN scheme) or the West of Scotland Specialist Virology Centre (HPS scheme) for molecular testing. Laboratory confirmation was undertaken using RT-PCR assays for circulating influenza $A$ viruses, influenza $B$ viruses and other respiratory viruses including respiratory syncytial virus and adenovirus [15-17].

\section{Statistical methods}

The two exposures of interest were vaccination with 2009/10 seasonal trivalent influenza vaccine and vaccination with either Pandemrix or Celvapan. Respiratory samples with a delay greater than 29 days between illness onset and sample collection were excluded as viral load is likely to be substantially reduced so long after disease onset. Although any such reduction in sensitivity (provided specificity remains high) is unlikely to affect VE estimates [18], a sensitivity analysis was undertaken restricting the VE estimation to a maximum of seven days between illness onset and sample collection. Only two individuals (both controls) had received a second dose of pandemic vaccine at the time of this study; these were not categorised differently to those who had received one dose.

Individuals were considered vaccinated if their date of seasonal or pandemic vaccination was 14 days or more before the date of onset [2]. As there is some evidence that the immune response induced by pandemic vaccines is more rapid than for seasonal vaccines ( $E$. Miller, HPA, personal communication), sensitivity analyses were carried out including individuals with a date of pandemic vaccination seven or more days before onset of symptoms.

For individuals whose date of onset was missing, the date of sample minus the median delay between illness onset and sample collection (three days) was assumed. As this assumption may affect the estimate of VE (if the exposure of interest is misclassified), we also investigated the effect of using the actual date of sample, or date of sample minus seven days for individuals with a missing date of onset. For the small number of samples (0.5\%) for which the date of sample collection was missing, the date of receipt in the laboratory was used instead.

VE was estimated using logistic regression models with pandemic influenza $A\left(\mathrm{H}_{1} \mathrm{~N}_{1}\right)_{2009} P C R$ result as outcome and seasonal or pandemic vaccination status as the linear predictor. VE can then be estimated as 1 -[odds ratio] [18]. Age (coded into five standard age groups, 45 years, 5-14 years, 15-44 years, 45-64 years and 65 years and above), sex, seasonal influenza vaccination status, country (England or Scotland), surveillance scheme (HPS, RCGP or RMN), date of sample collection (month) and the number of days delay between onset of symptoms and sample collection (coded into five categories: 0-1 day, 2-4 days, 5-7 days, 8-14 days and 15-29 days) were investigated as potential confounding variables.

Model selection for seasonal or pandemic VE estimation was performed by initially including age, date and vaccination status as covariates in the regression model. Other variables were added if they were significant and changed the vaccination odds ratios by $20 \%$ or more. Subgroup analyses by age group ( 15 years and $\geq 15$ years), for individuals who had received only 
one dose of vaccine, and for samples collected within seven days of onset were carried out.

As there were a large number of individuals with missing pandemic vaccination status, including only complete case data could potentially have lead to bias if the missing information was not completely at random. Instead, these observations were coded as 'vaccination status unknown' and included in the logistic regression models. The effect of excluding these individuals or classifying them as unvaccinated was also investigated. Individuals coded as vaccinated with pandemic vaccine, but with an unknown date of vaccination, were initially excluded from the logistic regression models. A sensitivity analysis was then carried out by refitting the final model assuming that those with missing vaccination dates for seasonal vaccine had all been vaccinated before 17 October (implying they would all have had an immune response by 1 November), and that those with missing pandemic vaccination dates had all been vaccinated on 21 October. We also investigated the effect of using week rather than month of sample collection as an indicator of time period. All statistical analyses were carried out in $\mathrm{R}$ version 2.10.1[19].

Vaccination status information collected on the swab request forms was validated by linking swab records from the HPS and RCGP swabbing schemes to electronic records from a subset of the practice team information database from HPS and electronic database records from RCGP network practices, respectively [20,21]. Linkage was achieved using age, sex, date of swab collection and practice post code for RCGP and the community health index ( $\mathrm{CHI}$ ) number for the HPS scheme. This also allowed an investigation of the vaccination status of persons with missing vaccination information on the swab request form. Validation was not possible for swabs collected through the RMN scheme.

\section{Ethics approval}

In England, ethics approval was not required and informed consent was not sought. The work was carried out under National Health Service (NHS) Act 2006 (section 251) for England, which provides statutory support for disclosure of such data by the NHS, and their processing by the HPA, for purposes of communicable disease control. In Scotland, ethics approval was not required and informed consent was not sought. HPS remains a constituent part of the NHS and coordinates the investigation and management of all national outbreaks.

\section{Results}

This report comprises information on 5,985 individuals whose samples were collected through the three surveillance systems in the study period, and who had a known PCR result. Two persons were positive for influenza $B$ and one other person was positive for influenza $A\left(H_{3}\right)$ : these three individuals were not included at any stage of the analysis. Of the remaining 5982, $1,746(29.2 \%)$ were positive for influenza $A\left(\mathrm{H}_{1} \mathrm{~N}_{1}\right), 630$ individuals (10.5\%) were positive for other respiratory viruses, and 3,606 individuals (60.3\%) were negative for all viruses tested. Table 1 shows the distribution and completeness of the baseline characteristics of the study participants according to whether they were cases or controls.

For the 663 individuals (11.1\%) for whom the date of onset was missing, the date of sample minus the median delay (three days) was used. The proportion with missing date of onset was not significantly higher among those positive for pandemic influenza $\mathrm{A}\left(\mathrm{H}_{1} \mathrm{~N}_{1}\right) 2009$ than among those who were negative: 174 of $1,746(10.0 \%)$ compared with 487 of $4,236(11.5 \%)$, chi-square test $p=0.09$. The proportion of individuals with unknown pandemic vaccination status (Table 1) was significantly higher among cases than controls (chi-square test $p<0.001$ ). The proportion of individuals with unknown pandemic vaccination status decreased between November $(1,982$ of 3,572 with unknown vaccination status, $55.5 \%$ ) and January (207 of $640,32.3 \%$ ).

Of the 186 individuals who had received pandemic vaccine, only two $(1.1 \%)$ had received two doses of vaccine: the remainder had received one dose of pandemic vaccine. Of the 97 vaccinated individuals for whom vaccine brand was known, only one had received Celvapan (one dose) and the rest Pandemrix.

One hundred and thirty individuals had received both seasonal and pandemic vaccines. This amounted to $69.9 \%$ of the 186 pandemic vaccinees and $21.6 \%$ of the 601 individuals who had received seasonal vaccination

\section{Pandemic vaccine effectiveness}

Among individuals who had received the pandemic vaccine, four of $85(4.7 \%)$ were positive for pandemic influenza $\mathrm{A}\left(\mathrm{H}_{1} \mathrm{~N}_{1}\right) 200914$ days after vaccination, compared with 870 (28.4\%) of 3,067 unvaccinated individuals who were positive. This difference was statistically significant (chi-square test $p<0.0001$ ), giving a crude pandemic VE estimate in preventing confirmed pandemic influenza $\mathrm{A}\left(\mathrm{H}_{1} \mathrm{~N}_{1}\right) 2009$ infection of $88 \%(95 \%$ confidence interval (Cl): $66 \%$ to $95 \%$ ).

The four vaccine failures occurred in people aged between 15 and 64 years. Three of them had received Pandemrix, and for one vaccine brand was unknown. All had received one dose.

The VE of the pandemic vaccine, adjusted for age group and sampling date (month) was $72 \%$ (95\% Cl: $21 \%$ to $90 \%$ ) (Table 2). These were the only two variables which altered the crude VE estimate by more than $20 \%$. As the vaccine failures all occurred in adults, the unadjusted pandemic VE point estimate in children aged less than 15 years was 100\% (binomial exact $95 \% \mathrm{Cl}$ : $74 \%$ to $100 \%$ ), and in adults aged 15 years and over, the pandemic VE estimate was $67 \%$ (95\% Cl: $6 \%$ to $88 \%$ ). 
Adjusted seasonal influenza VE was $-30 \%(95 \% \mathrm{Cl}$ : $-89 \%$ to $11 \%)$. This estimate was adjusted for age group, sampling date (month) and pandemic vaccination status; these were the only variables which were significantly associated with a positive test result for pandemic influenza $A\left(\mathrm{H}_{1} \mathrm{~N}_{1}\right)_{2009}$ and altered the crude odds ratio for seasonal influenza vaccination status by more than $\mathbf{2 0} \%$. If all individuals with an unknown date of seasonal influenza vaccination were assumed to be vaccinated on 17 October (and should therefore have developed protection by 1 November), the adjusted VE of the seasonal influenza vaccine was $-22 \%(95 \% \mathrm{Cl}$ : $-60 \%$ to $8 \%$ ).

As a number of individuals included with a missing date of onset $(n=616)$ were included in the final model, we examined the effect of setting the date of onset as equal to the date of sampling or date of sampling minus seven days if the date of onset was missing. The point estimates of the VE for either seasonal or pandemic vaccination remained the same. Several other sensitivity analyses were also carried out, with varying assumptions about the vaccination status of individuals with missing vaccination status (Table 2 ).

The adjusted VE estimate remained robust to varying assumptions about the true vaccination status and date of vaccination of individuals for whom this information was missing, and restriction to various subgroups. If vaccine protection was assumed to be induced after seven or more days rather than 14 days, 120 individuals could be classified as vaccinated with pandemic vaccine, among whom seven (5.8\%) were positive for pandemic influenza $\mathrm{A}\left(\mathrm{H}_{1} \mathrm{~N}_{1}\right)_{2009}$. This gave an adjusted pandemic VE estimate of $71 \%$ (95\% Cl: $37 \%$ to $87 \%$ ). There was only a minimal effect on VE when using week of sample collection rather than month (as a factor variable) in controlling for time period.

In order to validate data on pandemic vaccination status, RCGP and HPS swab data were linked to general practitioner (GP) records. Linkage was successful for a total of 1,468 individuals (of whom 910 were in the HPS scheme and 558 in the RCGP scheme). Of the 41 individuals recorded as vaccinated in the dataset from the swabbing programme, four (9.8\%) did not have a record of vaccination in GP databases; however vaccination could have occurred in a hospital setting. Among the 606 individuals who were unvaccinated according to the swabbing dataset, only two (0.3\%) were vaccinated according to the GP records and 604 were unvaccinated. Among the 821 individuals for whom there was no information on pandemic vaccination status in the swabbing dataset, only seven (0.9\%) were vaccinated according to their GP records, the rest (99.1\%) were unvaccinated. The proportion of vaccinated individuals in this group was significantly (chi-square test $p<0.001$ ) lower than among individuals with a known vaccination status, among whom $3.1 \%$ (95\% Cl: $2.7 \%$, to $3.6 \%$ ) were vaccinated (Table 1 ).

\section{Discussion}

This study has demonstrated high effectiveness of the newly developed monovalent pandemic influenza vaccine against confirmed pandemic influenza $\mathrm{A}\left(\mathrm{H}_{1} \mathrm{~N}_{1}\right) 2009$ infection one week after vaccination although the proportion of the study population that had received vaccination was low. No significant association, protective or otherwise, between trivalent seasonal influenza vaccination and confirmed pandemic influenza $A\left(\mathrm{H}_{1} \mathrm{~N}_{1}\right) 2009$ infection has been identified.

The case-control design employed in this study is an established method to estimate effectiveness of seasonal influenza vaccine in several countries [14, 22-26] and its robustness has been validated [21]. There are, however, potential limitations: Firstly, a convenience sample was used because random sampling of patients for a routine surveillance system based on GP-provided care is not feasible. It is unlikely, however, that the sampling would have caused substantial bias: although it is conceivable that a GP might selectively sample patients based on their vaccination status, their case or control status would not have been known at the time of sampling. Thus any selection bias would be randomly distributed. Selection bias could occur if severity of symptoms was related to influenza $\mathrm{A}\left(\mathrm{H}_{1} \mathrm{~N}_{1}\right)$ 2009-positive status, and GPs selectively sampled from persons with more severe symptoms whom they also know were vaccinated (although instructions are to sample the first few cases seen every week, regardless of vaccination status). This scenario would lead to an underestimation of VE. Secondly, as the vast majority of vaccinated individuals in this study for whom the vaccine brand was known had received Pandemrix, our results will not be applicable to Celvapan. Indeed, the study reflects the distribution of doses by vaccine brand delivered in the UK. Consequently, the estimated VE presented here is mainly applicable to Pandemrix. Thirdly, there were no data available on whether an individual had a chronic condition and therefore was in a target group for pandemic influenza vaccination. As the presence of a chronic condition may increase the severity of illness associated with influenza (compared to other respiratory infections) and thus the likelihood of seeking treatment in primary care, this may have lead to an underestimation of VE. A larger, more detailed study based on individual data from general practices would provide the possibility to adjust for such potential confounders. Fourthly, the impact of the influenza $A\left(\mathrm{H}_{1} \mathrm{~N}_{1}\right) 2009$ pandemic was greatest in children and young people, very few of whom had received the seasonal vaccine. For this reason, the effect of seasonal vaccination cannot be measured with precision. Finally, a number of samples lacked information on vaccination status. Several sensitivity analyses were carried out to examine the effect of various assumptions regarding vaccination status for those with missing vaccination status information. The pandemic VE estimates, however, appeared robust in these scenarios. Furthermore, validation of a sample of the RCGP and HPS swab data showed agreement of $99.1 \%$ between the information provided on the swab request form and 


\section{TABLE 1}

Personal and clinical characteristics of pandemic influenza A(H1N1) cases and controls, United Kingdom, 1 November 2009 - 29 January $2010(\mathrm{~N}=5,982)$

\begin{tabular}{|c|c|c|}
\hline Variable & Number of cases ( $\%$ of cases $N=1,746$ ) & Number of controls ( $\%$ of controls $N=4,236$ ) \\
\hline \multicolumn{3}{|l|}{ Received pandemic vaccine } \\
\hline Vaccinated $\geq 14$ days before onset & $4(0.2)$ & $81(1.9)$ \\
\hline Vaccinated 7-13 days before onset & $3(0.2)$ & $32(0.8)$ \\
\hline Vaccinated $<7$ days before onset & $10(0.6)$ & $45(1.1)$ \\
\hline Vaccinated - date unknown & o (o) & $11(0.3)$ \\
\hline Unvaccinated $^{a}$ & $877(50.2)$ & $2,225(52.5)$ \\
\hline Vaccination status unknown & $852(48.8)$ & $1,842(43.5)$ \\
\hline \multicolumn{3}{|l|}{ Received seasonal vaccine } \\
\hline Vaccinated $\geq 14$ days before onset & $52(3.0)$ & $234(5.5)$ \\
\hline Vaccinated $\ll 14$ days before onset & $15(0.9)$ & $85(2.0)$ \\
\hline Vaccinated - date unknown & $45(2.6)$ & $170(4.0)$ \\
\hline Unvaccinated $^{a}$ & $1,476(84.5)$ & $3,313(78.2)$ \\
\hline Vaccination status unknown & $158(9.0)$ & $434(10.2)$ \\
\hline \multicolumn{3}{|l|}{ Sex } \\
\hline Female & $934(53.5)$ & $2,486(58.7)$ \\
\hline Male & $797(45.6)$ & $1,708(40.3)$ \\
\hline Unknown & $15(0.9)$ & $42(1.0)$ \\
\hline \multicolumn{3}{|l|}{ Age group (years) } \\
\hline$<5$ & $211(12.1)$ & $824(19 \cdot 5)$ \\
\hline $5-14$ & $597(34.2)$ & $550(13.0)$ \\
\hline $15-44$ & $723(41.4)$ & $1,790(42.3)$ \\
\hline $45-64$ & $192(11.0)$ & $790(18.6)$ \\
\hline $65+$ & $21(1.2)$ & $265(6.3)$ \\
\hline Unknown & $2(0.1)$ & $17(0.4)$ \\
\hline \multicolumn{3}{|l|}{ Date of sample } \\
\hline November 2009 & $1,308(74.9)$ & $1,399(33.0)$ \\
\hline December 2009 & $371(21.2)$ & $2,264(53.4)$ \\
\hline 1-29 January 2010 & $67(3.8)$ & $573(13.5)$ \\
\hline \multicolumn{3}{|c|}{$\begin{array}{l}\text { Interval (days between onset and sample col- } \\
\text { lection) }\end{array}$} \\
\hline $0-1$ & $384(22.0)$ & $616(14 \cdot 5)$ \\
\hline $2-4$ & $844(48.3)$ & $1,773(41.9)$ \\
\hline $5-7$ & $247(14.1)$ & $823(19.4)$ \\
\hline 8-14 & $72(4.1)$ & $378(8.9)$ \\
\hline $15-29$ & $17(1.0)$ & $110(2.6)$ \\
\hline$\geq 30$ & $8(0.5)$ & $47(1.1)$ \\
\hline Unknown & $174(10.0)$ & $489(11.5)$ \\
\hline \multicolumn{3}{|l|}{ Surveillance scheme } \\
\hline RCGP & $608(34.8)$ & $1,581(37.3)$ \\
\hline RMN & $186(10.7)$ & $548(12.9)$ \\
\hline HPS & $952(54.5)$ & $2,107(49.7)$ \\
\hline
\end{tabular}

HPS: Health Protection Scotland RCGP: Royal College of General Practitioners' surveillance scheme; RMN: Health Protection Agency (HPA) Regional Microbiology Network.

${ }^{a}$ By date of onset. 
the GP electronic record. The proportion of persons recorded as vaccinated by their GP was significantly lower among those with missing pandemic vaccination information on the swab request form compared to those where this information was available.

This study demonstrates that the pandemic influenza vaccine was highly effective in reducing confirmed pandemic influenza infection in persons consulting in primary care. In addition, it provides evidence of protection from as early as seven days after vaccination. This discovery corroborates findings of the high immunogenicity of pandemic vaccines in clinical trials: a UK study has reported that $79 \%$ of participants had seroconverted by 14 days after receiving a single dose of MF-59-adjuvanted vaccine [2]. More recent published work done after introduction of the pandemic vaccine into the German national programme has demonstrated it to be highly effective using the screening method [27]. However, although the investigators adjusted for the confounding effect of age, the screening method should be treated cautiously due to potential unrecognised confounding [28]. Our VE findings have been adjusted for various confounders. The results are similar to the estimated effectiveness of the traditional trivalent non-adjuvanted seasonal influenza vaccine during periods in which the vaccine is well matched with the circulating influenza strain $[26,29]$, and the pandemic VE estimated here is considerably higher than in seasons of vaccine mismatch [23].

The peak of pandemic influenza activity during the second wave was in October 2009, at which stage the pandemic vaccine programme had only just started. Thus only a small proportion of the eligible population had been vaccinated at a time when pandemic virus was circulating widely. Consequently, although the observed pandemic VE was high in this study, because uptake was relatively low at this stage, any impact of the programme on disease at the population level would be more limited. This highlights the challenge of rapidly developing a new vaccine and implementing a new vaccine programme.

This study found no evidence that vaccination with $2009 / 10$ trivalent seasonal influenza vaccine was associated with increased or decreased risk of subsequent pandemic influenza $A\left(\mathrm{H}_{1} \mathrm{~N}_{1}\right)_{2009}$ infection in the UK. This contrasts with conflicting published reports that seasonal influenza vaccine might either increase subsequent risk of pandemic influenza [11] or alternatively provide protection against pandemic influenza, particularly severe disease [7]. This study replicates findings from case-cohort studies in Australia and the United States, in which no protective effect was reported from the 2008/o9 seasonal vaccine $[8,9]$. This observation suggests that cross protection from earlier seasonal vaccination cannot be assumed.

In conclusion, this study provides evidence that the pandemic influenza $A\left(\mathrm{H}_{1} \mathrm{~N}_{1}\right)_{2009}$ vaccine provided good protection against infection with pandemic influenza $A\left(\mathrm{H}_{1} \mathrm{~N}_{1}\right) 2009$ seven days or more after vaccination during the pandemic period. Further work is required to ascertain the effectiveness of the pandemic vaccine in children, in specific clinical risk groups and by individual vaccine brand.

\section{Acknowledgements}

We are grateful to the many primary care physicians in England and Scotland who supplied the clinical information on their patients; to the staff of the HPA Respiratory Virus Unit, the HPA regional microbiology laboratories and the West of Scotland Specialist Virology Centre who undertook analysis of specimens. We thank the staff of the Health Protection Agency, RCGP and Health Protection Scotland teams who coordinate the GP schemes, in particular Hayley Durnall from the RCGP and Joy Field from the HPA; Catherine Frew from WoSSVC and Dr Alison Potts from HPS for overseeing data collection. We thank Professors Maria Zambon and Liz Miller for helpful comments and support.

\section{TABLE 2}

Adjusted pandemic vaccine effectiveness under various assumptions and exclusion criteria, United Kingdom, 1 November 2009 - 29 January 2010

\begin{tabular}{|l|c|}
\hline Assumption or exclusion criterion & $\begin{array}{c}\text { Adjusted pandemic vaccine effectiveness } \\
\text { (95\% confidence interval) }\end{array}$ \\
\hline $\begin{array}{l}\text { Individuals with missing vaccination dates excluded, individuals with missing } \\
\text { vaccination status included as separate category }\end{array}$ & $72 \%(21 \%-90 \%)$ \\
\hline All individuals with missing vaccination status are assumed unvaccinated & $71 \%(20 \%-90 \%)$ \\
\hline $\begin{array}{l}\text { All individuals with missing vaccination dates are assumed vaccinated on } 21 \\
\text { October }\end{array}$ & $74 \%(28 \%-91 \%)$ \\
\hline Including only those individuals who received one dose of vaccine & 5,808 \\
\hline Excluding individuals with missing pandemic vaccination status & $71 \%(20 \%-90 \%)$ \\
\hline $\begin{array}{l}\text { Excluding individuals with an interval between onset and sampling of more than } \\
\text { seven days }\end{array}$ & $73 \%(26 \%-90 \%)$ \\
\hline Pandemic vaccination protection begins after seven days & $70 \%(15 \%-89 \%)$ \\
\hline Using week rather than month as indicator of time period & 3,147 \\
\hline
\end{tabular}

adjusted for age group and sampling date (month). 
Funding

This work has been undertaken as part of a European Centre for Disease Prevention and Control (ECDC) funded project "I-MOVE", which is co-ordinated by Epi-Concept (http://www.epiconcept.fr/).

\section{Conflicts of interest}

All authors have completed the Unified Competing Interest form at http://www.icmje.org/coi_disclosure.pdf (available on request from the corresponding author) and declare that DMF has received funding to attend influenza related meetings and has received consultancy fees from influenza vaccine manufacturers who might have an interest in the submitted work in the previous there years. In addition, The Virus Reference Department of the Health Protection Agency receives funding from a variety of vaccine manufacturers who might have an interest in the submitted work. All other authors declare they have no conflicts of interest.

\section{References}

1. Dawood FS, Jain S, Finelli L, Shaw MW, Lindstrom S, Garten RJ, et al. Emergence of a novel swine-origin influenza $A\left(\mathrm{H}_{1} \mathrm{~N}_{1}\right)$ virus in humans. New Engl J Med 2009;360(25):2605-15.

2. Clark TW, Pareek M, Hoschler K, Dillon H, Nicholson KG, Groth $\mathrm{N}$, et al. Trial of 2009 influenza $A\left(\mathrm{H}_{1} \mathrm{~N}_{1}\right)$ monovalent MF59adjuvanted vaccine. N Engl J Med. 2009;361(25):2424-35.

3. Liang XF, Wang HQ, Wang JZ, Fang HH, Wu J, Zhu FC, et al. Safety and immunogenicity of 2009 pandemic influenza $A \mathrm{H}_{1} \mathrm{~N}_{1}$ vaccines in China: a multicentre, double-blind, randomised, placebo-controlled trial. Lancet. 2010;375(9708):56-66.

4. Plennevaux E, Sheldon E, Blatter M, Reeves-Hoche MK, Denis $M$. Immune response after a single vaccination against 2009 influenza $\mathrm{A} \mathrm{H}_{1} \mathrm{~N}_{1}$ in USA: a preliminary report of two randomised controlled phase 2 trials. Lancet. 2010;375(9708):41-8

5. Vajo Z, Tamas F, Sinka L, Jankovics I. Safety and immunogenicity of a 2009 pandemic influenza $A H_{1} N_{1}$ vaccine when administered alone or simultaneously with the seasonal influenza vaccine for the 2009-10 influenza season: a multicentre, randomised controlled trial. Lancet. 2010; 375(9708):49-55.

6. Zhu FC, Wang H, Fang HH, Yang JG, Lin XJ, Liang XF, et al. A novel influenza $A\left(\mathrm{H}_{1} \mathrm{~N}_{1}\right)$ vaccine in various age groups. $\mathrm{N}$ Engl J Med. 2009; 361(25):2414-23

7. Garcia-Garcia L, Valdespino-Gomez JL, Lazcano-Ponce E, Jimenez-Corona A, Higuera-Iglesias A, Cruz-Hervert P, et al. Partial protection of seasonal trivalent inactivated vaccine against novel pandemic influenza $A / \mathrm{H}_{1} \mathrm{~N}_{1}$ 2009: case-control study in Mexico City. BMJ. 2009;339:b3928.

8. Kelly H, Grant K. Interim analysis of pandemic influenza ( $\left.\mathrm{H}_{1} \mathrm{~N}_{1}\right)_{2009}$ in Australia: surveillance trends, age of infection and effectiveness of seasonal vaccination. Euro Surveill. 2009;14(31): pii=19288. Available from: http://www. eurosurveillance.org/ViewArticle.aspx?Articleld =19288

9. Centers for Disease Control and Prevention (CDC). Effectiveness of 2008-09 trivalent influenza vaccine against 2009 pandemic influenza A ( $\left.\mathrm{H}_{1} \mathrm{~N}_{1}\right)$ - United States, May-June 2009. MMWR Morb Mortal Wkly Rep. 2009;58(44):1241-5.

10. Carcione D, Giele C, Goggin LS, Kwan KS, Smith DW, Dowse GK, et al. Association between 2009 seasonal influenza vaccine and influenza-like illness during the 2009 pandemic: preliminary results of a large household transmission study in Western Australia. Euro Surveill. 2010;15(28): pii=19616. Available from: http://www.eurosurveillance.org/ViewArticle. aspx?Articleld=19616

11. Skowronski DM, De Serres G, Crowcroft NS, Janjua NZ, Boulianne N, Hottes TS, et al. Association between the 2008o9 Seasonal Influenza Vaccine and Pandemic H1N1 Illness during Spring-Summer 2009: Four Observational Studies from Canada. PLoS Med. 2010;7(4):e1000258.

12. Donaldson L, Beasley $\mathrm{C}$, Ridge $\mathrm{K}$. The $\mathrm{H}_{1} \mathrm{~N}_{1}$ swine flu vaccination programme 2009-2010. Dear colleague letter. London: Department of Health; 15 Oct 2009. Available from: http://www.dh.gov.uk/en/Publicationsandstatistics/ Lettersandcirculars/Dearcolleagueletters/DH_107169

13. Pandemic $\mathrm{H}_{1} \mathrm{~N}_{1}$ (2009) Influenza: Chief Medical Officer's Fortnightly Bulletin for Journalists. Issue 5. London: Department of Health; 15 Mar 2010. Available from: http:// www.dh.gov.uk/prod_consum_dh/groups/dh_digitalassets/ documents/digitalasset/dh_114261.pdf

14. Fleming DM, Andrews NJ, Elllis JS, Bermingham A, Sebastianpillai P, Elliot AJ, et al. Estimating Influenza vaccine effectiveness using routinely collected laboratory data. J Epidemiol Community Health. 2010;64(12):1062-7.

15. Ellis J, Curran M. Simultaneous molecular detection and confirmation of influenza $\mathrm{AH}_{5}$, with internal control. Methods Mol Biol. 2011;665:161-81.
16. Ellis J, Iturriza M, Allen R, Bermingham A, Brown K, Gray J, et al. Evaluation of four real-time PCR assays for detection of influenza $\mathrm{A}\left(\mathrm{H}_{1} \mathrm{~N}_{1}\right)$ v viruses. Euro Surveill. 2009;14(22):pii=19230. Available from: http://www. eurosurveillance.org/ViewArticle.aspx?Articleld=19230

17. Gunson R, Maclean A, Davies E, Bennett S, Miller R, Carman WF. Development of a multiplex real-time RT-PCR that allows universal detection of influenza $A$ viruses and simultaneous typing of influenza $\mathrm{A} / \mathrm{H}_{1} \mathrm{~N}_{1} / 2009$ virus. J Virol Methods. 2010;163(2):258-61.

18. Orenstein EW, De Serres G, Haber MJ, Shay DK, Bridges CB, Gargiullo $\mathrm{P}$, et al. Methodologic issues regarding the use of three observational study designs to assess influenza vaccine effectiveness. Int J Epidemiol. 2007;36(3):623-31.

19. R Development Core Team. R: A language and environment for statistical computing. Vienna: R Foundation for Statistical Computing; 2008. Available from: http://www.r-project.org/

20. RCGP Research and Surveillance Centre: Weekly Returns Service Annual Report 2008. London: Royal College of General Practitioners. [Accessed 11 Aug 2010]. Available from: http:// www.rcgp.org.uk/clinical_and_research/rsc/annual_reports. aspx

21. General Practice - Practice Team Information. Edinburgh: Information Services Division; 31 Aug 2010. Available from: http://www.isdscotland.org/isd/1044.html

22. Centers for Disease Control and Prevention (CDC). Interim within-season estimate of the effectiveness of trivalent inactivated influenza vaccine--Marshfield, Wisconsin, 2007-08 influenza season. MMWR Morb Mortal Wkly Rep. 2008;57(15):393-8.

23. Belongia EA, Kieke BA, Donahue JG, Greenlee RT, Balish $A$, Foust $A$, et al. Effectiveness of inactivated influenza vaccines varied substantially with antigenic match from the 2004-2005 season to the 2006-2007 season. J Infect Dis. 2009;199(2):159-67.

24. Kelly H, Carville K, Grant K, Jacoby P, Tran T, Barr I. Estimation of influenza vaccine effectiveness from routine surveillance data. PLoS One 2009;4(3):e5079.

25. Skowronski DM, Masaro C, Kwindt TL, Mak A, Petric M, Li Y, et al. Estimating vaccine effectiveness against laboratoryconfirmed influenza using a sentinel physician network: results from the 2005-2006 season of dual $A$ and $B$ vaccine mismatch in Canada. Vaccine. 2007;25(15):2842-51.

26. Skowronski DM, De Serres G, Dickinson J, Petric M, Mak $A$, Fonseca K, et al. Component-specific effectiveness of trivalent influenza vaccine as monitored through a sentinel surveillance network in Canada, 2006-2007. J Infect Dis. 2009;199(2):168-79.

27. Wichmann O, Stöcker P, Poggensee G, Altmann D, Walter $D$, Hellenbrand W, et al. Pandemic influenza $A\left(\mathrm{H}_{1} \mathrm{~N}_{1}\right) 2009$ breakthrough infections and estimates of vaccine effectiveness in Germany 2009-2010. Euro Surveill. 2010;15(18):pii=19561. Available from: http://www.eurosurveillance.org/ViewArticle. aspx?Articleld $=19561$

28. Farrington CP. Estimation of vaccine effectiveness using the screening method. Int J Epidemiol. 1993;22(4):742-6.

29. Bridges CB, Thompson WW, Meltzer MI, Reeve GR, Talamonti WJ, Cox NJ, et al. Effectiveness and cost-benefit of influenza vaccination of healthy working adults: A randomized controlled trial. JAMA. 2000;284(13):1655-63. 